\title{
Computed Tomography-Derived Skeletal Muscle Radiodensity Is an Early, Sensitive Marker of Age-Related Musculoskeletal Changes in Healthy Adults
}

\author{
Yeon Woo Jung ${ }^{1, *}$, Namki Hong ${ }^{2, *}$, Joon Chae $\mathrm{Na}^{3}$, Woong Kyu $\mathrm{Han}^{3}$, Yumie Rhee ${ }^{2}$ \\ ${ }^{1}$ Yonsei University College of Medicine; ${ }^{2}$ Division of Endocrinology, Endocrine Research Institute, Department of Internal \\ Medicine, ${ }^{3}$ Department of Urology, Yonsei University College of Medicine, Seoul, Korea
}

Background: A decrease in computed tomography (CT)-derived skeletal muscle radiodensity (SMD) reflects age-related ectopic fat infiltration of muscle, compromising muscle function and metabolism. We investigated the age-related trajectory of SMD and its association with vertebral trabecular bone density in healthy adults.

Methods: In a cohort of healthy adult kidney donors aged 19 to 69 years $(n=583)$, skeletal muscle index (SMI, skeletal muscle area/ height $^{2}$ ), SMD, and visceral-to-subcutaneous fat (V/S) ratio were analyzed at the level of L3 from preoperative CT scans. Low bone mass was defined as an L1 trabecular Hounsfield unit (HU) $<160 \mathrm{HU}$.

Results: L3SMD showed constant decline from the second decade (annual change $-0.38 \%$ and $-0.43 \%$ in men and women), whereas the decline of L3SMI became evident only after the fourth decade of life ( $-0.37 \%$ and $-0.18 \%$ in men and women). One HU decline in L3SMD was associated with elevated odds of low bone mass (adjusted odds ratio, 1.07; 95\% confidence interval, 1.02 to 1.13; $P=0.003$ ), independent of L3SMI, age, sex, and V/S ratio, with better discriminatory ability compared to L3SMI (area under the receiver-operating characteristics curve 0.68 vs. $0.53, P<0.001$ ). L3SMD improved the identification of low bone mass when added to age, sex, V/S ratio, and L3SMI (category-free net reclassification improvement $0.349, P<0.001$; integrated discrimination improvement $0.015, P=0.0165$ ).

Conclusion: L3SMD can be an early marker for age-related musculoskeletal changes showing linear decline throughout life from the second decade in healthy adults, with potential diagnostic value for individuals with low bone mass.

Keywords: Sarcopenia; Osteoporosis; Aging; Diagnostic screening programs; Computed tomography

\section{INTRODUCTION}

Sarcopenia and osteoporosis are becoming major public health issues in aging societies [1,2]. Sarcopenia is defined as age-re-

Received: 24 July 2021, Revised: 11 October 2021,

Accepted: 1 November 2021

Corresponding author: Yumie Rhee

Division of Endocrinology, Endocrine Research Institute, Department of Internal Medicine, Yonsei University College of Medicine, 50-1 Yonsei-ro,

Seodaemun-gu, Seoul 03722, Korea

Tel: +82-2-2228-1973, Fax: +82-2-392-5548, E-mail: yumie@yuhs.ac lated loss of muscle mass, strength, and physical performance [3]. Osteoporosis, can be similarly defined as the age-related decline in bone mass and strength [2]. Although separate, they are increasingly identified as co-occurring processes $[4,5]$. The

\section{Copyright $\odot 2021$ Korean Endocrine Society}

This is an Open Access article distributed under the terms of the Creative Commons Attribution Non-Commercial License (https://creativecommons.org/ licenses/by-nc/4.0/) which permits unrestricted non-commercial use, distribution, and reproduction in any medium, provided the original work is properly cited.

*These authors contributed equally to this work. 
association between these concurrent diseases has been widely reported, and growing evidence shows that bone and muscle are interacting tissues, not only at their anatomical interface, but also metabolically and mechanically [6-9].

For the diagnosis of sarcopenia, quantitative computed tomography (CT) is considered as a potential instrument to noninvasively determine muscle quantity [1]. Since CT can accurately differentiate between fat and muscle using the specific attenuation characteristics of each kind of tissue, it can provide accurate measurement of body composition with assessing quantitative and qualitative changes in muscle mass [10]. The cross-sectional area of skeletal muscle (SMA) at the level of the 3rd lumbar vertebra (L3) was endorsed to provide an accurate surrogate of the whole-body skeletal muscle mass using the recently updated guidelines of the European Working Group on Sarcopenia in Older People (EWGSOP 2) [1,11]. SMA has also been reported as a prognostic marker for several diseases including cancer $[12,13]$.

However, in addition to the muscle area, skeletal muscle radiodensity (SMD) reflects muscle composition including ectopic fat accumulation in the skeletal muscle, known as myosteatosis $[14,15]$. An age-related increase in fat accumulation within the skeletal muscle compromises muscle function and metabolic status $[14,16]$. Therefore, a decline in SMD may reflect age-related changes in skeletal muscle quality. However, the trajectory of SMD across aging and its association with low bone mass in healthy adults has not yet been thoroughly investigated.

In this study, we aimed to investigate the age-related trajectory of SMD and its association with vertebral trabecular bone mass in healthy adults.

\section{METHODS}

\section{Participants}

To analyze the age-related trajectory of CT-based body composition data derived from healthy adults, we retrospectively studied 601 healthy adult kidney donors between the ages of 19 and 69 years, who underwent CT scans at Severance Hospital (Seoul, Korea) as part of routine preoperative evaluation between 2005 and 2013 (Fig. 1). Kidney donors showed no evidence of type 1/2 diabetes mellitus, HIV infection, uncontrolled hypertension or a history of hypertension with evidence of end stage organ damage, nephrolithiasis (more than two episodes), sickle cell anemia, medically significant liver or heart disease, active or incompletely treated malignancy, acute symptomatic infection,
601 Healthy kidney donors, aged 19-69, with preoperative abdominal CT scan at Severance Hospital, Korea (2005-2013)

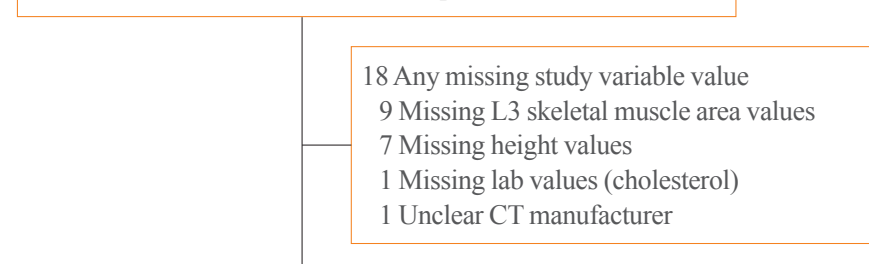

583 Participants who were finally selected for the analysis

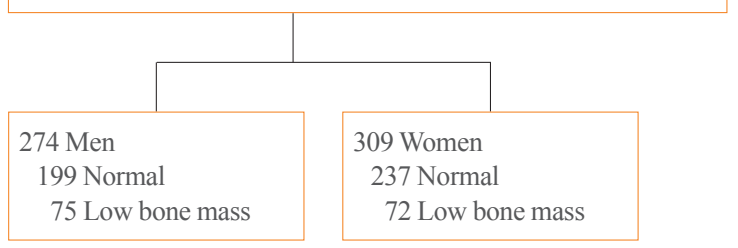

Fig. 1. Flow chart of the study. CT, computed tomography.

renal cystic disease, or psychiatric conditions requiring treatment before donation [17]. Participants were excluded if they had any of following conditions $(n=18)$ : missing L3 skeletal muscle area $(n=9)$; missing height $(n=7)$; missing laboratory values $(n=1)$; and unclear CT specification $(n=1)$. A total of 583 subjects were included in the final analyses. The study protocol was approved by the Institutional Review Board of Yonsei University Health System, Severance Hospital (IRB No. 4-2020-1154). The requirement for informed consent was waived owing to the retrospective nature of the medical record review.

\section{Measurements}

Laboratory tests were performed using fasting morning blood samples. The glomerular filtration rate (GFR) was estimated using the following Chronic Kidney Disease-Epidemiology equation: GFR $=141 \times \min (\mathrm{Scr} / \kappa, 1)^{\alpha} \times \max (\mathrm{Scr} / \kappa, 1)^{-1.209} \times 0.993^{\text {Age }} \times$ 1.018, where Scr is serum creatinine, $\kappa$ is 0.7 for females and 0.9 for males, $\alpha$ is -0.329 for females and -0.41 for males, $\min$ indicates the minimum $\mathrm{Scr} / \kappa, 1$, and max indicates the maximum $\mathrm{Scr} / \kappa, 1[18]$.

\section{Skeletal muscle, fat, and bone measurements from CT}

Quantification of skeletal muscle, bone, and fat area was performed using baseline $\mathrm{CT}$ scans obtained as part of the preoperative evaluation. Images were taken using multidetector CT scanners as follows: Sensation 64 (Siemens Healthcare, Forchheim, Germany) and SOMATOM Definition (Siemens Healthcare). The images utilized in this study were non-contrast abdominal CT with a slice thickness of $3 \mathrm{~mm}$, tube voltage of $120 \mathrm{kV}$, and exposure of $400 \mathrm{~mA}$ in the coronal plane. The datasets of body 
composition were reconstructed using a dedicated workstation (Aquarius iNtuition Edition version 4.4.12, TeraRecon, San Mateo, CA, USA) by a single analyst who was blinded to biochemical data and clinical outcomes. Measurement of skeletal muscle area (SMA, $\mathrm{cm}^{2}$ ), SMD (Hounsfield unit [HU]), visceral fat area $\left(\mathrm{VFA}, \mathrm{cm}^{2}\right)$, and subcutaneous fat area $\left(\mathrm{SFA}, \mathrm{cm}^{2}\right)$ was performed at the axial slice nearest to the lower edge of each lumbar vertebral body by applying predefined radiation attenuation. CT attenuation thresholds were -29 to $150 \mathrm{HU}$ for skeletal muscle, -190 to $-30 \mathrm{HU}$ for subcutaneous adipose tissue, and -50 to -150 HU for visceral adipose tissue [19]. Anterior abdominal muscles (external and internal obliques muscles, and rectus abdominus muscles), posterior abdominal muscles (psoas muscles, erector spinae muscles, quadratus lumborum muscles), and paraspinal muscles (erector spinae muscles and multifidus muscles), excluding intraabdominal visceral muscles, were considered as a region of interest (ROI) for SMA (Supplemental Fig. S1) [20]. Skeletal muscle index (SMI, $\mathrm{cm}^{2} / \mathrm{m}^{2}$ ) was calculated as SMA divided by height-squared to adjust for body size [21]. SMD was computed as the mean HU value of all pixels included in SMA. The workstation automatically traced the abdominal muscular wall to separate SFA and VFA, of which tissue boundaries were manually corrected, as necessary. To show the association of visceral fat with bone parameters after controlling for subcutaneous fat, we calculated the visceral-to-subcutaneous fat $(\mathrm{V} / \mathrm{S})$ ratio. To investigate inter- and intra-rater reliability, cross measurements of SMA, SMD, VFA, and SFA were conducted in 30 randomly selected participants between two analysts (Supplemental Table S1) $[22,23]$. All the intraclass correlation coefficient (ICC) values were over 0.98 , indicating good inter- and intra-rater reliability. Vertebral body attenuation at the L1 level was obtained using the simple trabecular ROI attenuation technique as suggested by Pickhardt et al. [24]. The simple trabecular ROI attenuation technique entails placing oval ROIs on the anterior trabecular region, avoiding the posterior venous plexus, surrounding cortical bone and any focal lytic or sclerotic lesion.

\section{Definition of sarcopenia and low bone mass}

Sex-specific mean and standard deviation (SD) values of SMA, SMI, and SMD at each lumbar vertebral level were calculated in healthy young adults aged 18 to 39 years (Supplemental Table S2). Sarcopenia (SMI) and sarcopenia (SMD) was defined as SMI or SMD at the level of $\mathrm{L} 3$ below $1 \mathrm{SD}$ of the young adult reference range (cutoff values: sarcopenia [SMI], 51.0 and $37.8 \mathrm{~cm}^{2} / \mathrm{m}^{2}$; sarcopenia [SMD], 42.3 and $36.9 \mathrm{HU}$ in men and women) (Supplemental Table S2) [25-27]. Lumbar spine trabecular attenua- tion in non-enhanced CT accurately predicted dual-energy X-ray absorptiometry (DXA) T-score-based osteoporosis [24]. Pickhardt et al. [24] suggested a threshold of $160 \mathrm{HU}$ for the mean coefficient of L1 trabecular bone attenuation to detect low bone mass with high sensitivity and specificity when using a standard $120 \mathrm{kV}$ single-energy CT scanner. Based on the study, low bone mass was defined as L1 trabecular bone attenuation $<160 \mathrm{HU}$.

\section{Statistical analysis}

All statistical tests were performed in $\mathrm{R}$ version 3.6.2 ( $\mathrm{R}$ Foundation for Statistical Computing, Vienna, Austria), using the package 'ggplot2' for data visualization and STATA 14.4 (Stata Corp., College Station, TX, USA). The characteristics of study subjects were described according to the presence of low bone mass. Variables were presented as mean $\pm \mathrm{SD}$, median (interquartile range), or as number (percentage). Differences in clinical characteristics were tested using two-sample independent $t$ tests, Wilcoxon rank-sum test, and chi-square test for continuous and categorical variables as appropriate. Age-related trajectories for SMI, SMA, SMD at the L3 level, trabecular bone attenuation at the L1 level, and VFA and V/S ratio at the L3 level were depicted based on a non-linear local polynomial regression model with a 95\% confidence interval (CI) using Stata's lpolyci command, which is a generalization of local mean smoothing as described by Nadaraya [28]. The association of L3SMI and L3SMD with low bone mass was evaluated using multivariable linear regression analyses, adjusted for age, sex, and V/S ratio. The ability to discriminate low bone mass between SMI and SMD was compared using receiver operating characteristics (ROC) curve analyses [29]. Net classification improvement (NRI) and integrated discrimination improvement (IDI) statistics were performed to assess the incremental discriminatory performance of L3SMD for detecting low bone mass when added to conventional predictors including SMI [30]. The statistical significance level was set at two-sided $P<0.05$.

\section{RESULTS}

\section{Baseline characteristics of study population}

A total of 583 kidney donor candidates were included in the analysis (mean age $39.3 \pm 11.2$ years, $53.0 \%$ females) (Table 1 ). Among the study population, $25.2 \%$ candidates $(n=147)$ had low bone mass according to the $\mathrm{L} 1$ trabecular bone attenuation cutoff of $160 \mathrm{HU}$ (men, $n=75$ [12.9\%]; women, $n=72$ [12.3\%], $P=0.301)$. The subjects with low bone mass had significantly lower SMA, SMD, and higher VFA at L3 in both men and 


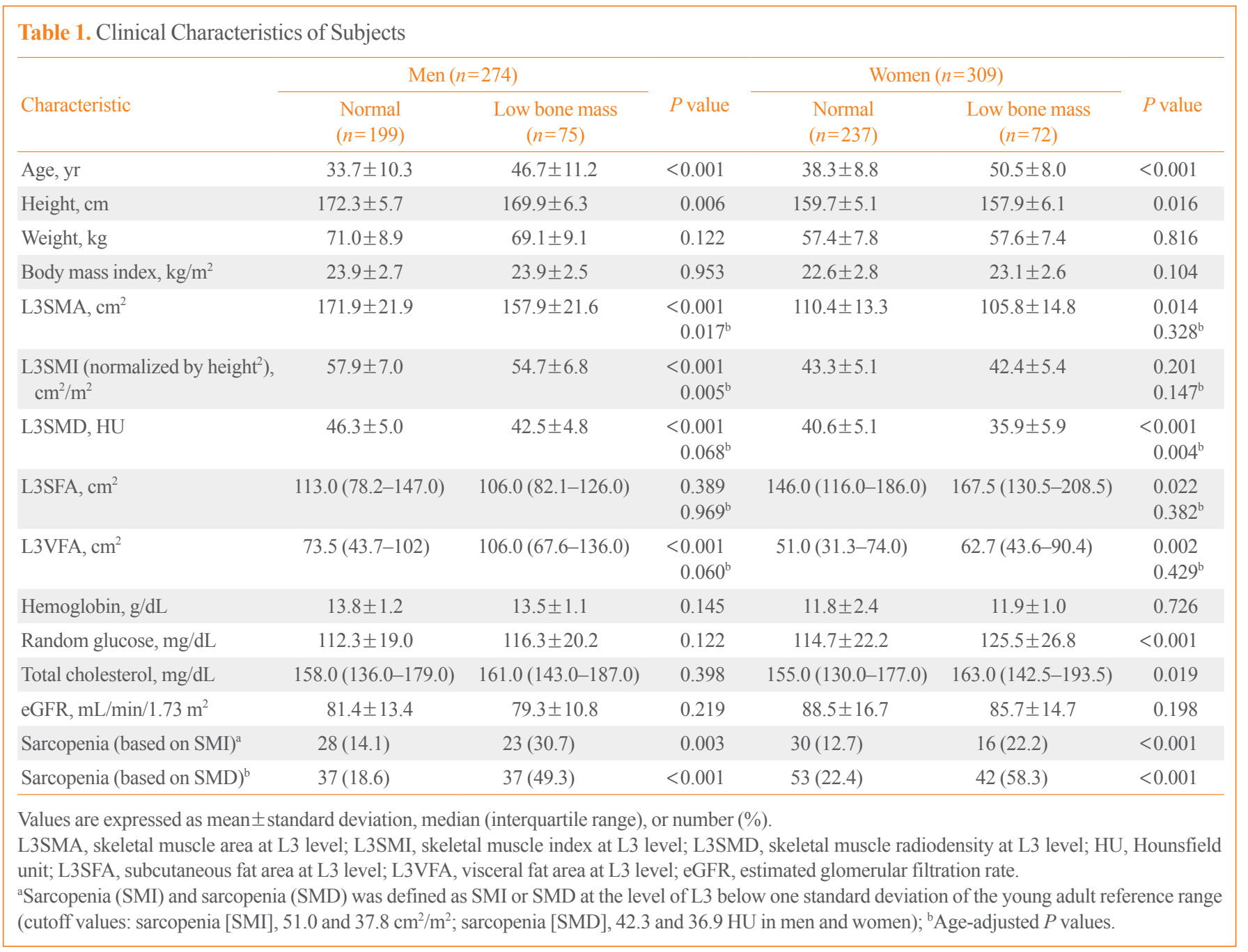

women. Overall, 97 subjects (16.6\%) had sarcopenia (SMI) and $169(29.0 \%)$ had sarcopenia (SMD). Subjects with low bone mass had a higher prevalence of sarcopenia (SMI) and sarcopenia (SMD) compared to those without low bone mass (men, $49.3 \%$ vs. $30.7 \%$; women, $58.3 \%$ vs. $22.2 \%$; $P<0.01$ for all).

\section{Age-related trends of skeletal muscle, fat, and bone parameters}

The age-dependent trajectories for the mean of L3SMD, L3SMI, L3SMA, L1 trabecular bone attenuation, VFA and V/S ratio are shown in Fig. 2, Supplemental Fig. S2. Age-related decline rate of L3SMA differed significantly before and after 40 years of age (men: $0.06 \%$ vs. $-0.53 \%, P=0.001$; women: $0.02 \%$ vs. $-0.31 \%$, $P=0.043$ ) (Supplemental Table S3), whereas L3SMD showed a constant decline rate without significant differences (men: $-0.50 \%$ vs. $-0.53 \%, P=0.833$; women: $-0.48 \%$ vs. $-0.47 \%, P=0.956$ ). When the prevalence of sarcopenia (SMI) and sarcopenia (SMD) was plotted against age groups by decade, the prevalence of sarcopenia (SMI) remained relatively constant with advancing age except for the sixth decade, whereas sarcopenia (SMD) showed a linear increasing trend across age from $8.1 \%$ to $70.2 \%$ ( $P$ for trend=0.016) (Fig. 3).

\section{L1 trabecular bone attenuation according to sarcopenia}

When subjects were grouped into combination of sarcopenia (SMD) and sarcopenia (SMI), L1 trabecular HU was lower in individuals with sarcopenia (SMD) in subgroups with (170 HU vs. $199 \mathrm{HU}, P<0.001$ ) or without sarcopenia (SMI) (145 HU vs. $192 \mathrm{HU}, P<0.001$ ) (Supplemental Fig. S3). Prevalence of low bone mass was increased in stepwise fashion from individuals without sarcopenia (SMD) and sarcopenia (SMI) toward those with both conditions ( $P$ for trend $<0.001$ ), with more prominent effect with presence of sarcopenia (SMD). 
L3SMI $\left(\mathrm{cm}^{2} / \mathrm{m}^{2}\right)$

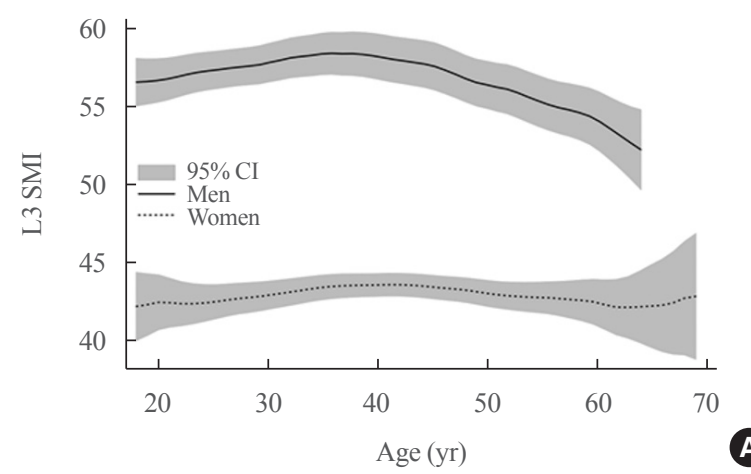

L1HU

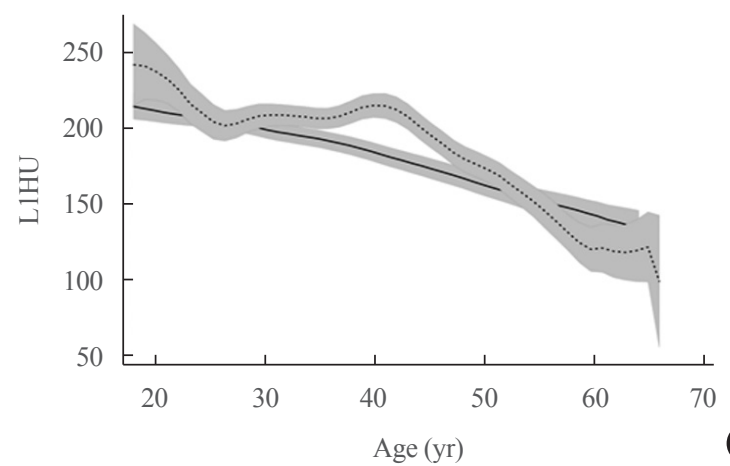

$\operatorname{L} 3 \operatorname{SMD}(\mathrm{HU})$

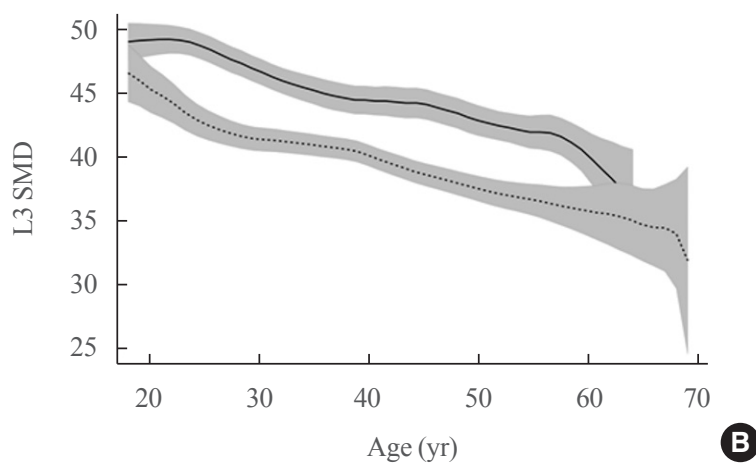

L3 V/S ratio

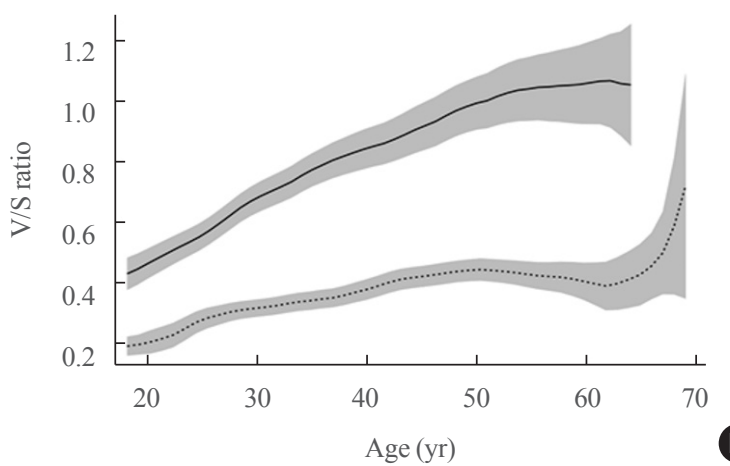

Fig. 2. Age-dependent trajectories for the mean of (A) skeletal muscle index at L3 level (L3SMI, $\mathrm{cm}^{2} / \mathrm{m}^{2}$ ), (B) skeletal muscle density at L3 level (L3SMD, Hounsfield unit [HU]), (C) vertebral bone attenuation at L1 level (L1HU, HU), and (D) visceral-to-subcutaneous fat ratio at L3 level (L3 V/S ratio). CI, confidence interval.
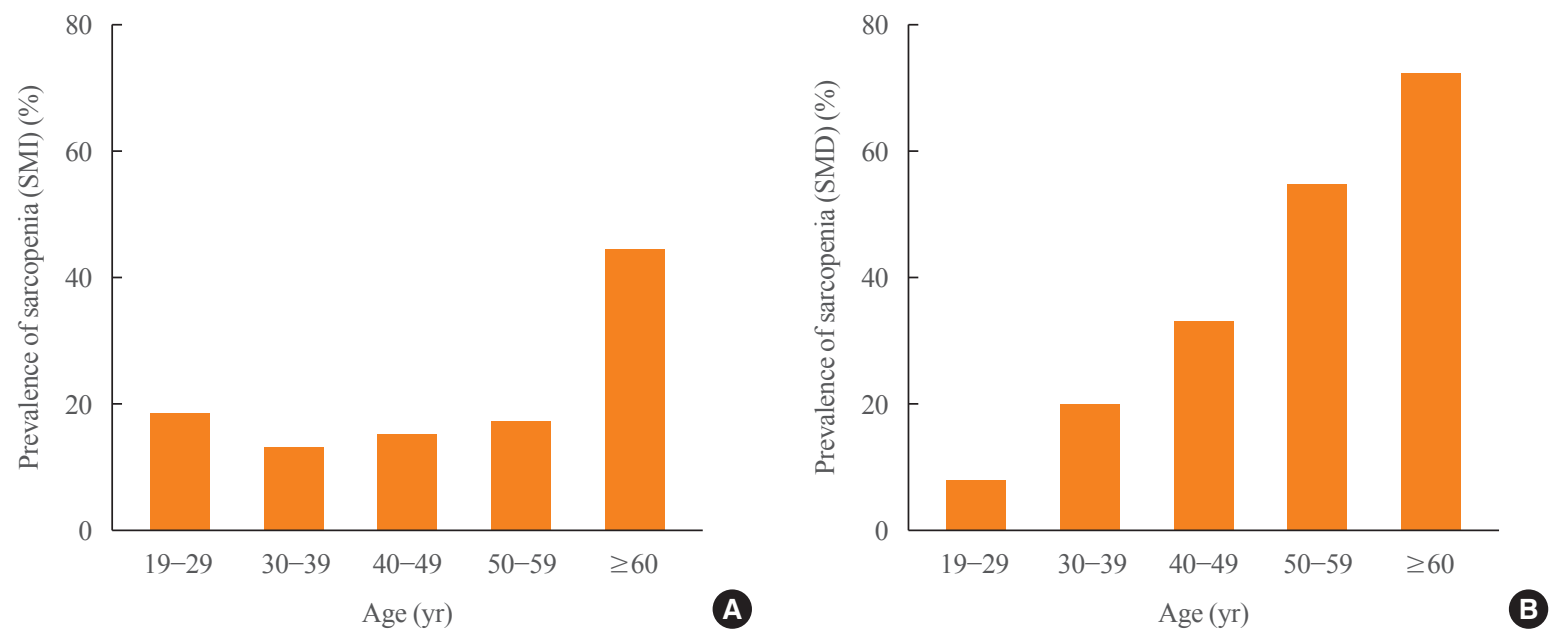

Fig. 3. The prevalence of population with normal muscle mass and sarcopenia based on skeletal muscle index (calculated as skeletal muscle area/height ${ }^{2}$ ) and skeletal muscle radiodensity is presented in percentage according to decade. (A) Sarcopenia (skeletal muscle index [SMI]) and (B) sarcopenia (skeletal muscle radiodensity [SMD]) was defined as SMI or SMD at L3 level below one standard deviation of young adult reference range (cutoff values: sarcopenia [SMI], 51.0 and $37.8 \mathrm{~cm}^{2} / \mathrm{m}^{2}$; sarcopenia [SMD], 42.3 and 36.9 Hounsfield unit [HU] in men and women). 
Table 2. Association of L3SMI and L3SMD with Low Bone Mass (Defined as L1HU < 160) in Healthy Kidney Donors

\begin{tabular}{|c|c|c|c|c|c|c|c|c|}
\hline \multirow[b]{2}{*}{ Variable } & \multicolumn{2}{|c|}{ Univariate model } & \multicolumn{2}{|c|}{ Multivariable model 1} & \multicolumn{2}{|c|}{ Multivariable model 2} & \multicolumn{2}{|c|}{ Multivariable model 3} \\
\hline & $\begin{array}{l}\text { Unadjusted OR } \\
(95 \% \mathrm{CI})\end{array}$ & $P$ value & $\begin{array}{c}\text { Adjusted OR } \\
(95 \% \mathrm{CI})\end{array}$ & $P$ value & $\begin{array}{c}\text { Adjusted OR } \\
(95 \% \mathrm{CI})\end{array}$ & $P$ value & $\begin{array}{c}\text { Adjusted OR } \\
(95 \% \mathrm{CI})\end{array}$ & $P$ value \\
\hline L3SMI, $/ 1 \mathrm{~cm}^{2} / \mathrm{m}^{2}$ decrease & $1.02(0.99-1.04)$ & 0.143 & $0.98(0.96-1.00)$ & 0.076 & $1.04(1.00-1.08)$ & 0.041 & $1.04(1.00-1.08)$ & 0.040 \\
\hline L3SMD, /1 HU decrease & $1.12(1.08-1.16)$ & $<0.001$ & $1.14(1.09-1.18)$ & $<0.001$ & $1.08(1.03-1.13)$ & 0.002 & $1.07(1.02-1.13)$ & 0.003 \\
\hline Age, $/ 1$ year increase & $1.14(1.11-1.16)$ & $<0.001$ & & & $1.12(1.09-1.15)$ & $<0.001$ & $1.12(1.09-1.15)$ & $<0.001$ \\
\hline Women (vs. men) & $0.81(0.55-1.17)$ & 0.259 & & & $0.20(0.10-0.41)$ & $<0.001$ & $0.26(0.11-0.59)$ & 0.001 \\
\hline $\mathrm{V} / \mathrm{S}$ ratio, $/ 0.1$ increase & $1.16(1.10-1.23)$ & $<0.001$ & & & & & $1.05(0.96-1.13)$ & 0.292 \\
\hline
\end{tabular}

L3SMI, skeletal muscle index at L3 level; L3SMD, skeletal muscle radiodensity at L3 level; L1HU, vertebral body attenuation at L1 level; OR, odds ratio; CI, confidence interval; HU, Hounsfield unit; V/S, visceral-to-subcutaneous fat.

\section{Association of L3SMD and L3SMI with low bone mass}

In univariate analysis, one HU decrement in L3SMD was associated with elevated odds of low bone mass (odds ratio [OR] $1.12, P<0.001$ ) (Table 2). In the multivariable models, the association of low L3SMD with low bone mass remained robust (adjusted odds ratio [aOR] 1.07 per $1 \mathrm{HU}$ decrement, $P=0.003$ ) independent of L3SMI (aOR, 1.04; $P=0.040$ ), age, sex, and V/S ratio. When grouped by sex, distinctive association between muscle parameters and low bone mass was observed that L3SMD (aOR, 1.10; $P=0.003$ ) but not L3SMI (aOR, 1.02; $P=0.651$ ) was the significant predictor of low bone mass in women, whereas L3SMI (aOR, 1.05; $P=0.030)$ remained as an independent predictor and statistical significance of L3SMD (aOR, 1.05; $P=$ 0.221) was attenuated in men (Supplemental Table S4). However, the effect modification for association between L3SMD and low bone mass by sex did not reach statistical significance $(P$ for interaction $=0.166$ for $\mathrm{L} 3 \mathrm{SMD} \times$ sex; $P$ for interaction $=0.522$ for L3SMI $\times$ sex). L3SMD predicts low bone mass better than L3SMI (area under the receiver-operating characteristics curve [AUC] 0.680 vs. $0.530 ; P<0.001$ ) (Supplemental Fig. S4). The discriminatory performance of the model to predict low bone mass was improved when L3SMD added to L3SMI (AUC, 0.530 to $0.693 ; P<0.001)$. AUC improvement by L3SMD did not reach statistical significance when added to the full model with L3SMI, age, sex, V/S ratio (AUC, 0.828 to $0.838 ; P=0.050$ ). However, NRI $(0.349 ; P<0.001)$ and IDI $(0.015 ; P=0.017)$ showed that L3SMD improved discrimination for low bone mass when added to the full model. When low SMD was entered into multivariable models as a categorical variable, the presence of sarcopenia (SMD) was associated with 1.9-fold elevated odds of low bone mass (aOR, 1.94; 95\% CI, 1.21 to 3.12; $P=0.006$ ), independent of sarcopenia (SMI) (aOR, 2.36; 95\% $\mathrm{CI}, 1.31$ to $4.25 ; P=0.004$ ), age, sex, and V/S ratio (Supplemen- tal Table S5). In subgroup analysis by sex (Supplemental Table S6), sarcopenia (SMD) remained significant predictor of low bone mass in women (aOR, 2.41; $P=0.011$ ), whereas statistical significance for sarcopenia (SMD) was attenuated in men (aOR, $1.63 ; P=0.169)$ without significant group interaction ( $P$ for interaction $=0.319$ ).

\section{DISCUSSION}

In this study, we investigated age-related trajectories for CT-derived skeletal muscle parameters including SMA, SMI, SMD at the level of L3 in healthy adult kidney donors. In the healthy kidney donor cohort, SMD showed a relatively linear decline from the early twenties throughout life, whereas the decline of SMI became evident after the fourth-to-fifth decade. Thus, SMD may play a role as an earlier, more sensitive marker representing age-related muscle changes. CT-derived SMD was also closely related to bone density. Individuals with low SMD values at L3 levels had elevated odds of low bone mass as measured by L1 trabecular bone attenuation, independent of L3SMI value, age, sex, and V/S ratio. L3SMD showed better discriminatory ability for the presence of low bone mass compared to L3SMI, with improved detection of low bone mass when added to covariates.

Earlier studies have showed that reduced skeletal muscle attenuation on CT was directly associated with ectopic fat accumulation within muscle, determined by biochemical and histological analysis, such that a lower attenuation reflects higher fat content [14,31]. Accumulation of intramyocellular fat was known to decrease insulin sensitivity, which can impair the capacity for normal protein synthesis in skeletal muscle, since insulin is anabolic to skeletal muscle $[32,33]$. Protein synthesis enhances muscle hypertrophy and the maintenance of muscle strength; thus, decreased insulin sensitivity can directly affect 
muscle mass and muscle strength [32]. Studies have suggested that ectopic fat infiltration in skeletal muscle is also related to functional deficits independent of muscle size by showing the correlation between thigh muscle attenuation and knee extension strength [34]. In our previous study, we observed that SMD also positively correlated with peak weight-corrected jump power in older adults [35]. Studies have increasingly demonstrated that a decline in muscle function started to manifest at an earlier age, compared to the concomitant loss of muscle mass $[3,36]$. Our findings showed the sensitive age-dependent decline of SMD, corresponding to the age-related increase of intramyocellular fat and decreased muscle function. These studies portent that SMD can be utilized as a surrogate marker for muscle quality, reflecting age-associated change in muscle.

This study showed that SMD at the third lumbar level could predict low bone mass independent of SMI as an indicator of muscle quantity. Additionally, SMD was a better predictor of low bone mass than SMI. Prior studies indicated that muscle function including muscle strength and physical performance had a strong relationship with bone mineral density and fracture risk [37-39]. This could be because the underlying mechanism of muscle deterioration and bone function that occurs with aging is accompanied by ectopic fat accumulation [32]. Aging is accompanied by accumulation of bone marrow adipocytes, and bone marrow fat accumulation is strongly associated with bone loss, suppressed bone formation, and increased fracture risk [32]. Apart from fatty infiltration of the bone marrow, fat accumulation in muscle has also been recognized as an important component of aging [40]. The association between SMD and low bone mass was robust after adjustment of the $\mathrm{V} / \mathrm{S}$ ratio, which can be interpreted as the decrease in muscle quality triggered by ectopic fat accumulation within the muscle. This might have a distinctive role on bone metabolism, independent of visceral or subcutaneous adiposity, which need to be validated further.

With the increasing utilization of CT in clinical practice, the concept of opportunistic $\mathrm{CT}$ (using the imaging modality beyond the primary diagnostic purpose) is emerging [41]. This approach allows for screening of low bone mass and sarcopenia without additional examination time, cost, or radiation [42]. Pickhardt et al. [24] suggested L1 trabecular bone attenuation thresholds to identify low BMD with $>90 \%$ sensitivity and specificity using screening thresholds of 160 and 110 HU. CT was typically used to evaluate muscle size by measuring muscle cross-section area on an axial CT image of L3, often with an adjustment for height, resulting in SMI [41]. Use of SMD may potentially improve the utility of CT by measuring skeletal muscle quality along with conventional measurements for muscle quantity [42]. A study based on CT scans of kidney donor provided cutoff for sarcopenia (SMD) as 2 SD below the average of population SMD values (34.3 and 34.8 HU for women and men, respectively) [26]. We modified this concept using $1 \mathrm{SD}$ below the average of L3 SMD values from young population aged 18 to 39 , yielded 36.9 $\mathrm{HU}$ for women and $42.3 \mathrm{HU}$ for men. A recent study based on young Korean population aged 20 to 44 suggested T-score approach for determining threshold for sarcopenia (SMD), grouped as class I ( $-1 \mathrm{SD}$ to $-2 \mathrm{SD}$; $45.9 \mathrm{HU}$ for women; 45.7 $\mathrm{HU}$ for men) and class II ( $-2 \mathrm{SD}$ or below; $39.9 \mathrm{HU}$ for women; $40.2 \mathrm{HU}$ for men) sarcopenia (SMD), which was similar to our approach [27]. However, thresholds for low SMD varied significantly among studies, at least partly due to potential variability in CT protocol and manufacturers across study centers. This needs to be addressed further in future studies to facilitate harmonized approach for utilizing SMD as a CT-derived biomarker.

This study has several limitations. Due to the retrospective, cross-sectional design of our study, the results do not reflect actual longitudinal changes in muscle attenuation with aging. Since this study was based on adult Korean kidney donors, our findings may not be generalizable to community-dwelling individuals or ethnically different individuals. However, our design enabled us to analyze CT scans from healthy donors across a wide age range, with a well-controlled CT protocol, which was optimal for examining the effect of aging on muscle changes without confounding diseases or medications. $\mathrm{CT}$ attenuation values, particularly $\mathrm{kVp}$, can be affected by $\mathrm{CT}$ manufacturers or scan protocols $[24,41]$. However, we only used CT scans with a fixed protocol of $120 \mathrm{kVp}$ and our findings were unchanged after further statistical adjustment for CT manufacturers in multivariable models. Since the present study only utilized CT-based definitions of sarcopenia and lacked collaborative information on muscle strength and physical performance, further investigation is needed to explore the association between CT-based muscle measurements and physical function. DXA-based osteoporosis, which is the current gold standard to define low bone mass, was not available in this study. However, we used L1 trabecular CT attenuation, a well validated marker that detects DXA-based osteoporosis with high sensitivity [24]. Although menopausal status is key determinant of bone and muscle health, menopausal status was not available in our dataset. Although we observed potential sexual dimorphism in association between muscle mass, quality, and bone mass, these findings need to be validated further in future studies with larger sample size. Serum 25-hydroxyvitamin D level, a potential confounder, was not available 
in this study. In this study, we were not able to measure body composition parameters using bioimpedance analysis (BIA) or whole body DXA. However, in our prior work, we found that skeletal muscle area at lumbar area showed substantial correlation ( 0.65 in women; $r=0.72$ in men) with appendicular lean mass measured using BIA [43]. Average body fat index in the waist $\left(\mathrm{cm}^{2} / \mathrm{kg}\right)$ derived from CT was highly correlated with body fat percent (\%) derived from BIA ( $r=0.82$ in women; $r=0.86$ in men).

In conclusion, CT-derived L3SMD can be an early, sensitive marker for age-related muscular changes, showing a linear decline throughout life from early in the second decade even in healthy adults. Low L3SMD was associated with elevated odds of low bone mass, independent of L3SMI, age, sex, and fat mass. Opportunistic screening based on L3SMD along with L3SMI from routine clinical CT may improve detection for individuals at high risk for sarcopenia, which merits further validation.

\section{CONFLICTS OF INTEREST}

No potential conflict of interest relevant to this article was reported.

\section{ACKNOWLEDGMENTS}

We thank the SENTINEL (Severance ENdocrinology daTa scIeNcE pLatform) project developed by Sung-Kil Lim Research Award (4-2018-1215) and the 2020 Research fund of the Department of Internal Medicine, Severance Hospital, Seoul, Korea. This work was supported by the Student Research grant of Yonsei University College of Medicine (Yeon Woo Jung, March 2020), faculty research grant of Yonsei University College of Medicine (6-2019-0102; Namki Hong), and the Korean Endocrine Society for New Faculty Convergence Research Award 2019 (Namki Hong). The abstract of this study was presented as poster at annual meeting of American Society for Bone and Mineral Research 2019.

\section{AUTHOR CONTRIBUTIONS}

Conception or design: Y.W.J., N.H. Acquisition, analysis, or interpretation of data: Y.W.J., N.H., J.C.N., W.K.H., Y.R. Drafting the work or revising: Y.W.J., N.H., J.C.N., W.K.H., Y.R. Final approval of the manuscript: Y.W.J., N.H., J.C.N., W.K.H., Y.R.

\section{ORCID}

Yeon Woo Jung https://orcid.org/0000-0001-9059-3628

Namki Hong https://orcid.org/0000-0002-8246-1956

Yumie Rhee https://orcid.org/0000-0003-4227-5638

\section{REFERENCES}

1. Cruz-Jentoft AJ, Bahat G, Bauer J, Boirie Y, Bruyere O, Cederholm T, et al. Sarcopenia: revised European consensus on definition and diagnosis. Age Ageing 2019;48:16-31.

2. World Health Organization. Assessment of fracture risk and its application to screening for postmenopausal osteoporosis: report of a WHO study group [meeting held in Rome from 22 to 25 June 1992] [Internet]. Geneva: WHO; 1994 [cited 2021 Nov 18]. Available from: https://apps.who.int/iris/handle/10665/39142.

3. Goodpaster BH, Park SW, Harris TB, Kritchevsky SB, Nevitt M, Schwartz AV, et al. The loss of skeletal muscle strength, mass, and quality in older adults: the health, aging and body composition study. J Gerontol A Biol Sci Med Sci 2006;61: 1059-64.

4. Yoo JI, Ha YC. Review of epidemiology, diagnosis, and treatment of osteosarcopenia in Korea. J Bone Metab 2018; 25:1-7.

5. Dao T, Green AE, Kim YA, Bae SJ, Ha KT, Gariani K, et al. Sarcopenia and muscle aging: a brief overview. Endocrinol Metab (Seoul) 2020;35:716-32.

6. Drey M, Sieber CC, Bertsch T, Bauer JM, Schmidmaier R; FiAT intervention group. Osteosarcopenia is more than sarcopenia and osteopenia alone. Aging Clin Exp Res 2016;28: 895-9.

7. Huo YR, Suriyaarachchi P, Gomez F, Curcio CL, Boersma $\mathrm{D}$, Gunawardene $\mathrm{P}$, et al. Comprehensive nutritional status in sarco-osteoporotic older fallers. J Nutr Health Aging 2015; 19:474-80.

8. Kawao N, Kaji H. Interactions between muscle tissues and bone metabolism. J Cell Biochem 2015;116:687-95.

9. Di Monaco M, Vallero F, Di Monaco R, Tappero R. Prevalence of sarcopenia and its association with osteoporosis in 313 older women following a hip fracture. Arch Gerontol Geriatr 2011;52:71-4.

10. Guerri S, Mercatelli D, Aparisi Gomez MP, Napoli A, Battista G, Guglielmi G, et al. Quantitative imaging techniques for the assessment of osteoporosis and sarcopenia. Quant Imaging Med Surg 2018;8:60-85. 
11. Shen W, Punyanitya M, Wang Z, Gallagher D, St-Onge MP, Albu J, et al. Total body skeletal muscle and adipose tissue volumes: estimation from a single abdominal cross-sectional image. J Appl Physiol (1985) 2004;97:2333-8.

12. Hopkins JJ, Reif RL, Bigam DL, Baracos VE, Eurich DT, Sawyer MB. The impact of muscle and adipose tissue on long-term survival in patients with stage I to III colorectal cancer. Dis Colon Rectum 2019;62:549-60.

13. Caan BJ, Cespedes Feliciano EM, Prado CM, Alexeeff S, Kroenke $\mathrm{CH}$, Bradshaw $\mathrm{P}$, et al. Association of muscle and adiposity measured by computed tomography with survival in patients with nonmetastatic breast cancer. JAMA Oncol 2018;4:798-804.

14. Goodpaster BH, Kelley DE, Thaete FL, He J, Ross R. Skeletal muscle attenuation determined by computed tomography is associated with skeletal muscle lipid content. J Appl Physiol (1985) 2000;89:104-10.

15. Miljkovic I, Zmuda JM. Epidemiology of myosteatosis. Curr Opin Clin Nutr Metab Care 2010;13:260-4.

16. Goodpaster BH, Krishnaswami S, Harris TB, Katsiaras A, Kritchevsky SB, Simonsick EM, et al. Obesity, regional body fat distribution, and the metabolic syndrome in older men and women. Arch Intern Med 2005;165:777-83.

17. Lentine KL, Kasiske BL, Levey AS, Adams PL, Alberu J, Bakr MA, et al. KDIGO clinical practice guideline on the evaluation and care of living kidney donors. Transplantation 2017;101(8S Suppl 1):S1-109.

18. Levey AS, Stevens LA, Schmid CH, Zhang YL, Castro AF 3rd, Feldman HI, et al. A new equation to estimate glomerular filtration rate. Ann Intern Med 2009;150:604-12.

19. Mourtzakis M, Prado CM, Lieffers JR, Reiman T, McCargar LJ, Baracos VE. A practical and precise approach to quantification of body composition in cancer patients using computed tomography images acquired during routine care. Appl Physiol Nutr Metab 2008;33:997-1006.

20. Lenchik L, Lenoir KM, Tan J, Boutin RD, Callahan KE, Kritchevsky SB, et al. Opportunistic measurement of skeletal muscle size and muscle attenuation on computed tomography predicts 1-year mortality in medicare patients. J Gerontol A Biol Sci Med Sci 2019;74:1063-9.

21. Heymsfield SB, Heo M, Thomas D, Pietrobelli A. Scaling of body composition to height: relevance to height-normalized indexes. Am J Clin Nutr 2011;93:736-40.

22. Schuck P. Assessing reproducibility for interval data in healthrelated quality of life questionnaires: which coefficient should be used? Qual Life Res 2004;13:571-86.
23. Koo TK, Li MY. A guideline of selecting and reporting intraclass correlation coefficients for reliability research. J Chiropr Med 2016;15:155-63.

24. Pickhardt PJ, Lee LJ, del Rio AM, Lauder T, Bruce RJ, Summers RM, et al. Simultaneous screening for osteoporosis at CT colonography: bone mineral density assessment using MDCT attenuation techniques compared with the DXA reference standard. J Bone Miner Res 2011;26:2194-203.

25. Janssen I, Heymsfield SB, Ross R. Low relative skeletal muscle mass (sarcopenia) in older persons is associated with functional impairment and physical disability. J Am Geriatr Soc 2002;50:889-96.

26. Derstine BA, Holcombe SA, Ross BE, Wang NC, Su GL, Wang SC. Skeletal muscle cutoff values for sarcopenia diagnosis using T10 to L5 measurements in a healthy US population. Sci Rep 2018;8:11369.

27. Kim HK, Kim KW, Kim EH, Lee MJ, Bae SJ, Ko Y, et al. Age-related changes in muscle quality and development of diagnostic cutoff points for myosteatosis in lumbar skeletal muscles measured by CT scan. Clin Nutr 2021;40:4022-8.

28. Nadaraya EA. On estimating regression. Theory Probab Appl 1964;9:141-2.

29. DeLong ER, DeLong DM, Clarke-Pearson DL. Comparing the areas under two or more correlated receiver operating characteristic curves: a nonparametric approach. Biometrics 1988;44:837-45.

30. Pencina MJ, D’Agostino RB Sr, D’Agostino RB Jr, Vasan RS. Evaluating the added predictive ability of a new marker: from area under the ROC curve to reclassification and beyond. Stat Med 2008;27:157-72.

31. Boutin RD, Yao L, Canter RJ, Lenchik L. Sarcopenia: current concepts and imaging implications. AJR Am J Roentgenol 2015;205:W255-66.

32. Hamrick MW, McGee-Lawrence ME, Frechette DM. Fatty infiltration of skeletal muscle: mechanisms and comparisons with bone marrow adiposity. Front Endocrinol (Lausanne) 2016;7:69.

33. Hong N, Lee J, Ku CR, Han K, Lee CR, Kang SW, et al. Changes of computed tomography-based body composition after adrenalectomy in patients with endogenous hypercortisolism. Clin Endocrinol (Oxf) 2019;90:267-76.

34. Goodpaster BH, Carlson CL, Visser M, Kelley DE, Scherzinger A, Harris TB, et al. Attenuation of skeletal muscle and strength in the elderly: the Health ABC Study. J Appl Physiol (1985) 2001;90:2157-65.

35. Choi H, Hong N, Park N, Kim CO, Kim HC, Choi JY, et al. 
Computed tomography-derived skeletal muscle radiodensity predicts peak weight-corrected jump power in older adults: the Korean Urban Rural Elderly (KURE) Study. Calcif Tissue Int 2021;108:764-74.

36. Alajlouni D, Bliuc D, Tran T, Eisman JA, Nguyen TV, Center JR. Decline in muscle strength and performance predicts fracture risk in elderly women and men. J Clin Endocrinol Metab 2020;105:dgaa414.

37. Proctor DN, Melton LJ, Khosla S, Crowson CS, O’Connor MK, Riggs BL. Relative influence of physical activity, muscle mass and strength on bone density. Osteoporos Int 2000; 11:944-52.

38. Blain H, Vuillemin A, Teissier A, Hanesse B, Guillemin F, Jeandel C. Influence of muscle strength and body weight and composition on regional bone mineral density in healthy women aged 60 years and over. Gerontology 2001;47:207-12.

39. Cawthon PM, Fullman RL, Marshall L, Mackey DC, Fink HA, Cauley JA, et al. Physical performance and risk of hip fractures in older men. J Bone Miner Res 2008;23:1037-44.

40. Figueiredo P, Marques EA, Gudnason V, Lang T, Sigurdsson S, Jonsson PV, et al. Computed tomography-based skeletal muscle and adipose tissue attenuation: variations by age, sex, and muscle. Exp Gerontol 2021;149:111306.

41. Yoon JK, Lee S, Kim KW, Lee JE, Hwang JA, Park T, et al. Reference values for skeletal muscle mass at the third lumbar vertebral level measured by computed tomography in a healthy Korean population. Endocrinol Metab (Seoul) 2021; 36:672-7.

42. Boutin RD, Lenchik L. Value-added opportunistic CT: insights into osteoporosis and sarcopenia. AJR Am J Roentgenol 2020;215:582-94.

43. Lee YS, Hong N, Witanto JN, Choi YR, Park J, Decazes P, et al. Deep neural network for automatic volumetric segmentation of whole-body CT images for body composition assessment. Clin Nutr 2021;40:5038-46. 